\title{
Composição química e estabilidade dimensional da madeira de três eucaliptos
}

\author{
Chemical composition and dimensional stability of \\ three eucalypts wood
}

Aline Krolow Soares ${ }^{1}$, Tainise Vergara Lourençon ${ }^{2}$, Rafael de Avila Delucis ${ }^{3}$, Darci Alberto Gatto ${ }^{1,3}$

\footnotetext{
${ }^{1}$ UFPel - Universidade Federal de Pelotas, Centro de Desenvolvimento Tecnológico (CDTec), Rua Gomes Carneiro, n.1, CEP: 96010-610 - Campus Anglo, Pelotas, Rio Grande do Sul, Brasil.

${ }^{2}$ Department of Bioproducts and Biosystems, Aalto University, Espoo, Finland.

${ }^{3}$ UFPel - Universidade Federal de Pelotas, Centro de Engenharias (CEng), Rua Benjamin Constant - CEP: 96010-020 Campus Cotada, Pelotas, Rio Grande do Sul, Brasil.

e-mail: alinekrolowsoares@yahoo.com.br, tainise@gmail.com,rdelucis@ hotmail.com,darcigatto@yahoo.com
}

\section{RESUMO}

Nesse estudo foram feitas correlações entre composição química e estabilidade dimensional das madeiras de Eucalyptus botryoides, Eucalyptus paniculata e Eucalyptus tereticornis. Para isso, foram confeccionados corpos de prova em cinco diferentes posições ao longo do perfil radial, correspondentes a 0, 25, 50, 75 e $100 \%$ de distância da medula. Para a análise física, os corpos de prova foram submersos em água até a saturação, condicionados sob temperatura de $20^{\circ} \mathrm{C}$ com umidade relativa (UR) de $65 \%$ e secos em estufa $\left(103 \pm 2^{\circ} \mathrm{C}\right)$. Foram coletados dados de massa e dimensões desses corpos de prova em cada uma dessas etapas, para posterior cálculo da densidade aparente a $12 \%$ e das contrações tangencial, radial e volumétrica. Para as análises químicas, amostras referentes a cada posição foram caracterizadas por meio dos teores de extrativos solúveis em etanol-tolueno, lignina Klason, hemicelulose e $\alpha$-celulose. Observou-se que as madeiras dos três eucaliptos apresentaram comportamento similar. O percentual de lignina e a densidade básica apresentaram correlação positiva, ambos aumentando no sentido medula-casca, enquanto a hemicelulose correlacionou-se negativamente com a densidade e o percentual de extrativos negativamente com os parâmetros de retratibilidade.

Palavras-chave: Eucalyptus, retratibilidade, química da madeira.

\begin{abstract}
In this study correlations between chemical composition and dimensional stability of Eucalyptus botryoides, Eucalyptus paniculata e Eucalyptus tereticornis were evaluated. Specimens were prepared from five different positions in radial direction, corresponding to $0,25,50,75$ e $100 \%$, from pith to bark. For physical analysis, samples mass and dimensions were measured above saturation point, at conditioned temperature $\left(20^{\circ} \mathrm{C}\right.$ and $65 \%$ of relative humidity) and oven dried $\left(103 \pm 2{ }^{\circ} \mathrm{C}\right)$. In each step density at $12 \%(\rho b)$, as well tangential $(\beta \mathrm{T})$, radial $(\beta \mathrm{R})$ and volumetric $(\beta \mathrm{V})$ shrinkage were calculated. Samples from same positions were chemical evaluated concerning to soluble extractives in ethanol-toluene, insoluble lignin, hemicellulose and $\alpha$-cellulose. It has been observed similar behavior among eucalypt wood. Lignin and density showed positive correlation, both increasing from pith to bark, while hemicellulose presented negative correlation with density and extractives negative correlation with shrinkage.
\end{abstract}

Keywords: Eucalyptus, shrinkage, wood chemistry.

\section{INTRODUÇÃO}

As primeiras espécies do gênero Eucalyptus foram implantadas no Brasil em meados da década de 60, para suprir a demanda da indústria ferroviária por dormentes e carvão vegetal [1]. Atualmente, a madeira de eucalipto é a principal matéria-prima da indústria brasileira de processamento de toras e tem aplicação em todo setor madeireiro. Porém, apesar do rápido crescimento, é normalmente abatida cedo, quando há maior proporção de lenho juvenil. 
Conforme $\mathrm{Wu}$ et al. [2], o lenho juvenil possui menor densidade e rigidez que o lenho adulto. Além disso, o lenho juvenil comparado ao adulto apresenta também variações relacionadas a aspectos mecânicos [3] e em menor grau variações químicas [4]. Afora as diferenças evidenciadas na comparação entre distintas espécies e lenhos, as características químicas e físicas da madeira apresentam variabilidade ao longo do perfil radial do tronco [5] [6] [7] [8].

Na composição química da madeira encontram-se a celulose e as hemiceluloses, polissacarídeos de estrutura semicristalina que sustentam a parede celular, e a lignina, composto aromático, tridimensional, que na planta funciona no transporte de água e nutrientes dentro da estrutura vascular e confere sustentação e resistência [9]. Além dos componentes macromoleculares responsáveis pela composição da parede celular, os extrativos possuem baixo peso molecular e são tipicamente associados à biodegradabilidade, cor e flamabilidade da madeira [10] [11].

As propriedades físicas estão mais fortemente relacionadas a qualidade da madeira. Dentre os principais comportamentos da madeira tidos como indesejáveis, diversos autores afirmam que a estabilidade dimensional é uma das principais causas de desperdícios de recursos no processamento da madeira, especialmente espécies de rápido crescimento [12]. A estabilidade dimensional da madeira pode ser caracterizada pela contração ou inchamento da madeira, quando em contato com a água, e está relacionada à capacidade da madeira em absorver água higroscópica [13] [14]. Esse mecanismo ocorre devido às interações existentes entre moléculas de água e hidroxilas presentes nos polissacarídeos da madeira, através das ligações de hidrogênio [15].

Embora existam diversos trabalhos avaliando propriedades físicas no sentido radial, bem como características tecnológicas tipicamente associadas à influência de propriedades químicas, poucos trabalhos foram direcionados nas correlações detalhadas entre propriedades físicas e químicas da madeira [16] [17]. Nesse contexto, o presente estudo teve como objetivo avaliar a correlação entre os parâmetros químicos e físicos de madeiras de rápido crescimento do gênero Eucalyptus, as quais são a principal matéria-prima da indústria madeireira.

\section{MATERIAIS E MÉTODOS}

A madeira utilizada nesse estudo foi proveniente de plantios homogêneos de Eucalyptus botryoides, Eucalyptus paniculata e Eucalyptus tereticornis, com aproximadamente 60 anos de idade, localizados no município de Charqueadas, sul do Brasil. Cinco árvores adultas de cada espécie, com diâmetro a altura do peito (DAP) superior a $50 \mathrm{~cm}$, foram selecionadas conforme o procedimento ASTM D5536 [18].

Foram extraídos discos no DAP com $8 \mathrm{~cm}$ de espessura de cada árvore, a partir dos quais foram retiradas baguetas centrais com $2 \mathrm{~cm}$ de largura, rejeitando-se a medula. Após esses procedimentos, amostras foram retiradas nas posições radiais $0,25,50,75$ e 100\%, as quais foram divididas de acordo com os dois lados da medula. Um dos lados destinou-se aos ensaios físicos e o outro às análises químicas (Figura 1).

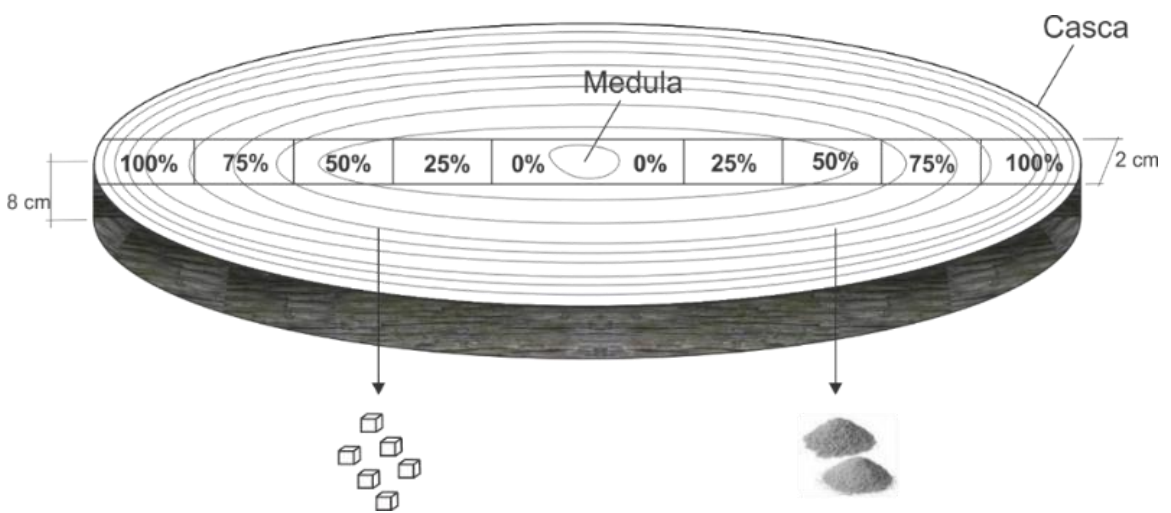

Figura 1: Esquema de confecção das amostras para as análises física e química.

Para mensuração das propriedades físicas, as amostras foram submersas em água até a saturação. Em seguida, foram alocadas em câmara climática $\left(20^{\circ} \mathrm{C}\right.$ de temperatura e $65 \%$ de umidade relativa do ar) até a estabilização do teor de umidade. Posteriormente, as amostras foram secas em estufa a $103 \pm 2{ }^{\circ} \mathrm{C}$ até massa constante. Após cada uma dessas etapas (saturação, climatização e secagem), foram coletados os dados de 
massa (balança digital com precisão de $0,01 \mathrm{~g}$ ) e dimensões (paquímetro digital com precisão de $0,01 \mathrm{~mm}$ ), os quais foram empregados no cálculo dos parâmetros físicos: densidade aparente a $12 \%(\rho)$, contrações radial $\left(\beta_{\mathrm{R}}\right)$, tangencial $\left(\beta_{\mathrm{T}}\right)$ e volumétrica $\left(\beta_{\mathrm{V}}\right)$ (Equações 1, 2 e 3 ).

$$
\begin{gathered}
\rho=\frac{\mathrm{m}_{12 \%}}{\mathrm{~V}_{12 \%}} * 100 \\
\beta_{\mathrm{R} ; \mathrm{T}}=\frac{\left(\mathrm{L}_{\mathrm{i}}-\mathrm{L}_{0}\right)}{\mathrm{L}_{\mathrm{i}}} * 100 \\
\beta_{\mathrm{V}}=\frac{\left(\mathrm{V}_{\mathrm{i}}-\mathrm{V}_{0}\right)}{\mathrm{V}_{\mathrm{i}}} * 100
\end{gathered}
$$

Em que: $\rho=$ densidade aparente à $12 \%\left(\mathrm{~g} . \mathrm{cm}^{-3}\right) ; \mathrm{m} 12 \%=$ massa a $12 \%$ de umidade da madeira $(\mathrm{g}) ; \mathrm{v} 12 \%=$ volume a $12 \%$ de umidade da madeira; $\beta=$ contração dimensional $(\%) ; \mathrm{R}=$ radial; $\mathrm{T}=$ tangencial; $\mathrm{V}=$ volumétrica; $\mathrm{L} 0=$ Dimensão da amostra seca no $(\mathrm{mm}) ; \mathrm{Li}=$ Dimensão inicial da amostra saturada $(\mathrm{mm}) ; \mathrm{V}_{0}=$ Volume da amostra seca $\left(\mathrm{mm}^{3}\right) ; \mathrm{Vi}=$ Volume da amostra saturada $\left(\mathrm{mm}^{3}\right)$.

Para as análises químicas, amostras referentes a cada posição, foram moídas em moinho do tipo Wiley, coletando-se o material retido na peneira de 60 mesh. Assim, foram quantificados o teor de extrativos solúveis em etanol:tolueno (E\%) (TAPPI T204 cm-97) [19], lignina insolúvel em ácido (L\%) (TAPPI T222 om-98) [20], hemicelulose ( $\mathrm{H} \%)$ e $\alpha$-celulose $(\mathrm{C} \%)$ [21], totalizando a holocelulose $(\mathrm{HO} \%)$. As relações entre as variáveis foram aferidas de acordo com o coeficiente de correlação de Pearson, que serviu como base para a comparação dos valores médios entre as posições radiais de cada espécie.

\section{RESULTADOS E DISCUSSÃO}

Ao correlacionar as propriedades químicas e físicas das madeiras de Eucaliptus (Tabela 1), pode-se observar que o percentual de $\mathrm{L} \%$ apresentou correlação positiva com a $\rho$ e negativa com as contrações tangencial e volumétrica. Nesse sentido, os maiores níveis de $\rho$ e L\% assim como as menores contrações, foram encontrados nas regiões de lenho adulto, próximas à casca (Figura 2).

Conforme Panda [22], a maior densidade no lenho adulto deve-se à espessura da parede celular ser maior que no lenho juvenil, a parede celular do lenho adulto tem menor atividade fisiológica por ser mais lignificada, explicando a proporção entre $\rho$ e $\mathrm{L} \%$ nos lenhos juvenil e adulto. Além disso, as menores contrações nestas regiões de maior densidade também foram observadas em um estudo com Corymbia citriodora de 50 anos de idade [8]. De acordo com Meylan [23], as maiores contrações em zonas menos densificadas do lenho ocorrem em razão dos maiores ângulos microfibrilares associados à essas regiões, os quais ocasionam um maior espaço inter-microfibrilar. 
Tabela 1: Coeficientes de correlação para as propriedades físicas e químicas para as madeiras de Eucalyptus.

\begin{tabular}{|c|c|c|c|c|c|c|c|c|c|}
\hline & $\rho$ & $\beta_{\mathrm{R}}$ & $\beta_{\mathrm{T}}$ & $\beta_{\mathrm{V}}$ & $\mathrm{L} \%$ & $\mathrm{C} \%$ & $\mathrm{H} \%$ & $\mathrm{HO} \%$ & $\mathrm{E} \%$ \\
\hline$\rho$ & 1 & $-0,19$ & $-0,33$ & $-0,27$ & $0,66^{*}$ & $-0,30$ & $-0,29$ & $-0,64^{*}$ & 0,48 \\
\hline$\beta_{\mathrm{R}}$ & & 1 & $0,79^{*}$ & $0,94 *$ & $-0,39$ & 0,35 & 0,03 & 0,44 & $-0,74 *$ \\
\hline$\beta_{\mathrm{T}}$ & & & 1 & $0,93 *$ & $-0,65^{*}$ & 0,31 & 0,16 & $0,52 *$ & $-0,75^{*}$ \\
\hline$\beta_{\mathrm{V}}$ & & & & 1 & $-0,55^{*}$ & 0,35 & 0,14 & $0,55^{*}$ & $-0,79 *$ \\
\hline $\mathrm{L} \%$ & & & & & 1 & $-0,32$ & $-0,17$ & $-0,55^{*}$ & $0,65^{*}$ \\
\hline $\mathrm{C} \%$ & & & & & & 1 & $-0,59 *$ & $0,54 *$ & $-0,63^{*}$ \\
\hline $\mathrm{H} \%$ & & & & & & & 1 & 0,36 & 0,03 \\
\hline $\mathrm{HO} \%$ & & & & & & & & 1 & $-0,70^{*}$ \\
\hline $\mathrm{E} \%$ & & & & & & & & & 1 \\
\hline
\end{tabular}

Em que: Valores seguidos por $*$ foram significativos com $5 \%$ de probabilidade de erro.

Eucalyptus tereticornis

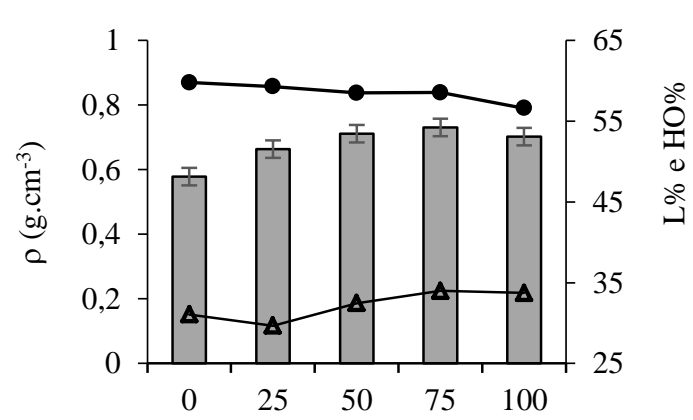

Posição radial (\%)
Eucalyptus paniculata

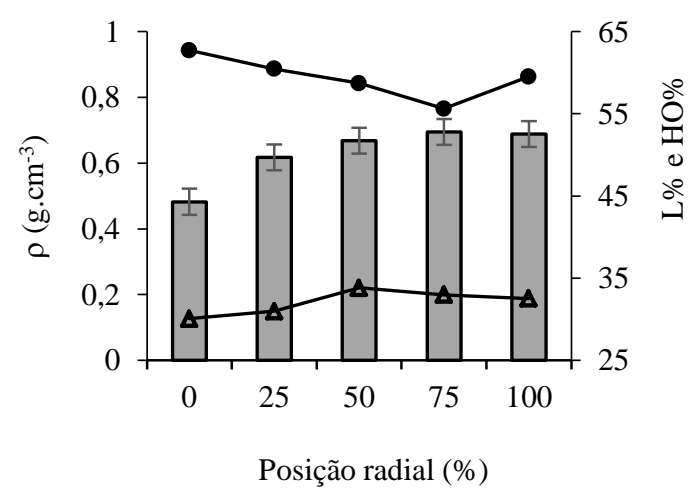

Eucalyptus botryoides

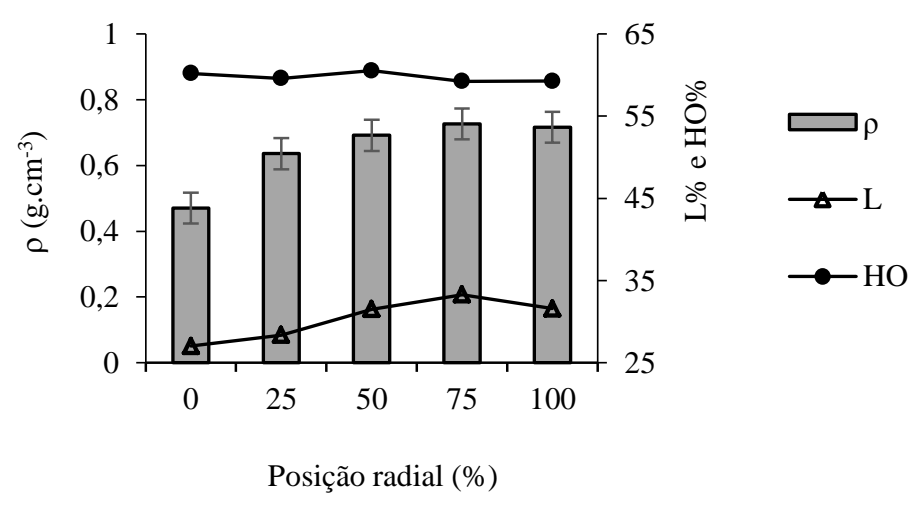

Figura 2: Relações entre densidade básica a $12 \%$ e teores de holocelulose e lignina das três espécies estudadas.

Ao contrário do percentual de L\%, o teor de holocelulose (HO\%) apresentou correlação negativa com 
$\rho$ e positiva com $\beta \mathrm{T}$ e $\beta \mathrm{V}$. Tais correlações de $\mathrm{HO} \%$ com propriedades físicas da madeira, opostas as correlações obtidas para L\%, foram resultados esperados, uma vez que os teores de lignina e holocelulose devem ser inversamente proporcionais, fechando o balanço mássico dos compostos macromoleculares da madeira.

Dessa forma, os teores de $\mathrm{HO} \%$ foram menores nas regiões próximas à casca (Figura 2), onde há maior proporção de lenho adulto. Assim, nas regiões mais próximas a medula, onde encontra-se o lenho juvenil, tem-se menor $\rho$ e maior $\mathrm{HO} \%$. A menor $\rho$ nessa região, além de estar relacionada com a anatomia da madeira (paredes mais delgadas e maior lúmen) [24] [25], pode ser associada a menor proporção de lignina e maior conteúdo de holocelulose nessa região. A holocelulose é uma soma de carboidratos, os quais apresentam menor peso molecular quando comparados com a lignina [26].

Com relação ao teor de E\%, verificou-se correlação negativa com todas as contrações (Tabela 1). Assim, enquanto a contração da madeira foi menor nas posições próximas à casca, ainda que ligeiramente, $o$ teor de E\% mostrou-se superior, em comparação com as primeiras posições a partir da medula (Figura 3).

Nesse contexto, as maiores contrações próximas a medula já relacionadas com a menor densidade e menor parede celular do lenho, também podem estar relacionadas aos extrativos, menos abundantes nessa região. Conforme Barbosa et al. [27], os extrativos têm a capacidade de interagir com as moléculas de água, interferindo nos mecanismos de inchamento e contração da madeira.

Assim, regiões com maiores quantidades de extrativos tendem a ser as mesmas que apresentam menor contração e inchamento, tendo em vista que os extrativos hidrofóbicos impedem a absorção e desorção de água da parede celular, devido à falta de afinidade química com a água [28] [29] [30].

Eucalyptus tereticornis

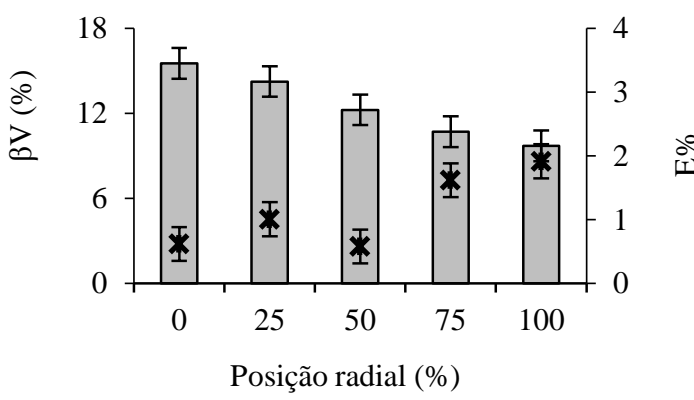

Eucalyptus paniculata

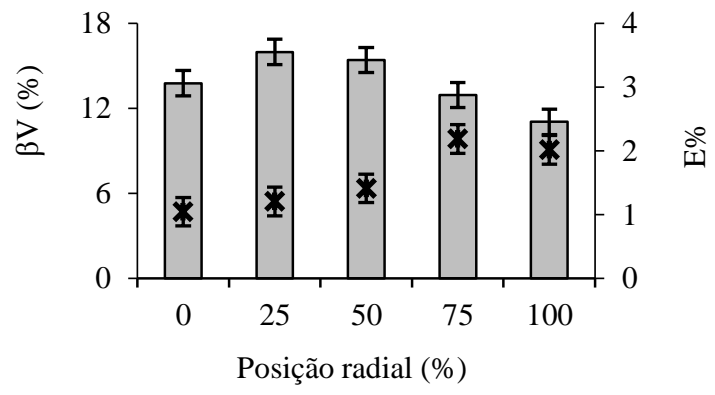

Eucalyptus botryoides

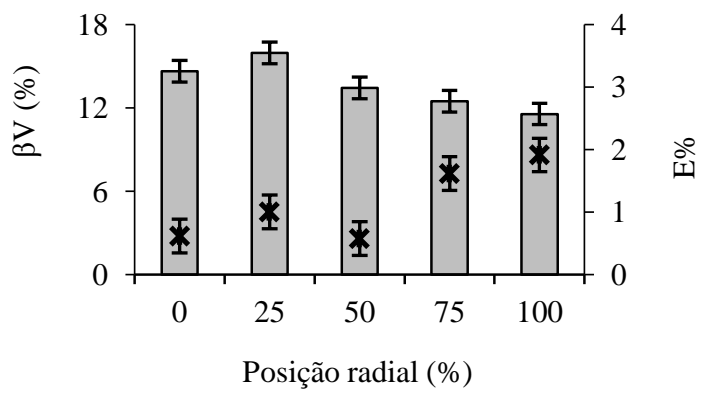

Figura 3: Relações do teor de extrativos e contração das três espécies estudadas.

Ainda que os extrativos hidrofílicos tenham a capacidade de interagir com moléculas de água e interferir na contração da madeira, os baixos percentuais presentes nessas espécies (entre 0,5 e $2 \%$ ) indicam que as contrações estão mais associadas com características anatômicas da madeira. De acordo com Chagas et al. [17], a espessura e o diâmetro de fibra são as principais características anatômicas que influenciam nas propriedades físicas. 
Quando comparados entre as três espécies, os valores médios de $\rho$, L\% e HO\% encontrados mostraram-se semelhantes entre si (Figura 2), estando de acordo com a faixa de valores de $\rho$ (entre 0,39 e 0,87 g.cm-3), L\% (entre 20,0 e 31,0\%) e HO\% (entre 63,8 e 74,4\%) descritos na literatura para espécies do mesmo gênero [5] [6] [8] [31] [32]. Da mesma forma, os valores médios para $\beta V$ (entre 13,07 e 28,69\%) e E\% (entre 1,7 e 2,7\%) encontrados neste estudo estão de acordo com a literatura para as espécies do gênero Eucalyptus [5] [13] [27] [32].

\section{CONCLUSÕES}

Nesse trabalho foram quantificados os componentes químicos de madeiras de Eucalyptus botryoides, Eucalyptus paniculata e Eucalyptus tereticornis e correlacionados com suas propriedades físicas, na direção radial do lenho. As propriedades químicas e físicas das madeiras de eucalipto apresentaram uma variação radial semelhante. Os valores de $\mathrm{L} \%$ e $\mathrm{HO} \%$ apresentaram correlações com $\rho, \beta \mathrm{T}$ e $\beta \mathrm{V}$. Na medida em que $\rho$ aumentou (sentido medula-casca), L\% também teve acréscimo, enquanto $\mathrm{HO} \%$ e retratibilidade reduziram. Isso indica que o teor de $\mathrm{L} \%$ presente diminui a contração da madeira, impedindo a absorção de água. O teor de E\% mostrou-se maior nas regiões de menor contração (lenho adulto). Isso indica que o teor de E\% pode ter influência nas propriedades físicas de contração para a madeira do gênero Eucaliptus.

\section{AGRADECIMENTOS}

Os autores gostariam de agradecer à Fundação de Amparo à Pesquisa do Rio Grande do Sul (FAPERGS), ao Conselho Nacional de Desenvolvimento Científico e Tecnológico (CNPq), e à Coordenação de Aperfeiçoamento de Pessoal de Nível Superior (CAPES), processo 00.889.834/0001.08, pelo suporte financeiro.

\section{BIBLIOGRAFIA}

[1] SOUZA, J. T., TREVISAN, R., DENARDI, L., et al., "Qualidade da madeira serrada proveniente de árvores dominantes e médias de Eucalyptus grandis submetidas à secagem”, Cerne, v. 18, n. 1, pp. 167-174, 2012.

[2] WU, H. X., ELDRIDGE, K. G., MATHESON, A. C., et al., "Achievement in forest tree improvement in Australia and New Zealand 8. Successful introduction and breeding of radiata pine to Australia", Australian Forestry, v. 70, n. 4, pp. 215-225, 2007.

[3] VIDAURRE, G., LOMBARDI, L.R., OLIVEIRA, J.T.D.S., et al., "Lenho Juvenil e Adulto e as Propriedades da Madeira", Floresta e Ambiente, v.18, pp.469-480, 2011.

[4] BAO, F.C., JIANG, Z.H., JIANG, X.M., et al., "Differences in wood properties between juvenile wood and mature wood in 10 species grown in China", Wood Science and Technology, v.35, pp. 363-375, 2001.

[5] OLIVEIRA, J. T. S., SILVA, J. C. "Variação radial da retratibilidade e densidade básica da madeira de Eucalyptus saligna Sm.”, Revista Árvore, v. 27, n. 3, pp. 381-385, 2003.

[6] SEVEro, E. T. D., CAlOnEGO, F. W., SAnSÍGOlO, C. A. "Composição química da madeira de Eucalyptus citriodora em função das direcções estruturais”, Silva Lusitana, v. 14, n. 1, pp. 113-126, 2006.

[7] VALENTE, B. M. R. T., EVANGELISTA, W. V., SILVA, J. S., et al., "Avaliação radial e longitudinal das propriedades mecânicas e da massa específica básica da madeira de angico-vermelho (Anadenanthera peregrina (L.) Speg.)", Scientia Fororestalis, v. 39, n. 92, pp. 463-468, 2011.

[8] LOURENÇON, T., GATTO, D., MATTOS, B., et al., "Propriedades físicas da madeira de Corymbia citriodora no sentido radial", Scientia Forestalis, v. 41, n. 99, pp. 369-375, 2013.

[9] FENGEL, D., WEGENER, G. "Wood Chemistry, Ultrastructure, Reactions", Berlin/New York: Walter de Gruyter, 2003.

[10] CHANG, T. C., CHANG, H. T., WU, C. L., et al., "Influences of extractives on the photodegradation of wood", Polymer Degradation and Stability, v. 95, n. 0, pp. 516-521, 2010.

[11] POLETTO, M. "Effect of extractive content on the thermal stability of two wood species from Brazil", Maderas. Ciencia y Tecnología, v. 18, pp. 435-442, 2016.

[12] MANHIÇA, A. A., ROCHA, M. P., JUNIOR, R. T. "Eficiência operacional no desdobro de Pinus utilizando modelos de corte numa serraria de pequeno porte", Cerne, v.19, n.2, pp.339-346, 2013. 
[13] BATISTA, D. C., KLITZKE, R. J., SANTOS, C. V. T. "Densidade básica e retratibilidade da madeira de clones de três espécies de Eucalyptus" Ciência Florestal, v. 20, n. 4, pp. 665-674, 2010.

[14] DELUCIS, R. A., GATTO, D. A., STANGERLIN, D. M., et al., "Segregação e variação radial das propriedades físicas da madeira juvenil e adulta de cedro (Cedrela fissilis Vellozo)", Scientia Florestalis, v. 41, n. 100, pp. 549-556, 2013.

[15] OLIVEIRA, J. T. S., FILHO, M. T., FIEDLER, N. C. "Avaliação da retratibilidade da madeira de sete espécies de Eucalyptus”, Revista Árvore, v. 34, n. 5, pp. 929-936, 2010.

[16] VALE, A. T., DIAS, I. S., SANTANA, M. A. E. "Relações entre propriedades químicas, físicas e energéticas da madeira de cinco espécies de cerrado", Ciência Florestal, v. 20, n. 1, pp. 137-145, 2010.

[17] CHAGAS, S. F., EVANGELISTA, W. V., SILVA, J. C., et al., "Propriedades da madeira de teca de diferentes idades e oriundas de desbaste", Ciência da Madeira, v. 5, n. 2, pp. 138-150, 2014.

[18] ASTM - AMERICAN SOCIETY FOR TESTING AND MATERIALS. D5536-94: "Standard methods of testing small clear specimens of timber", Philadelphia, 2010.

[19] TAPPI, Solvent Extractives of Wood and Pulp. T. T204 cm-97, "Technical Association of the Pulp and Paper Industry", Atlanta, USA, 1997.

[20] TAPPI, Acid-insoluble lignin in wood and pulp. T222 om-98, "Technical Association of the Pulp and Paper Industry", Atlanta, USA, 1998.

[21] ROWELL, R. M. "Handbook of Wood Chemistry and Wood Composites", Second Edition. Boca Raton: Taylor \& Francys, 2012.

[22] PANDA, I. "Handbook on Coal, Lignin, Wood and Rosin Processing", Chapter 5: Degradation of wood. Delhi, India, 2016.

[23] MEYLAN, B. A. "The influence of microfibril angle on the longitudinal shirinkage-moisture content relationship", Wood Science and Technology, v. 6, n. 4, pp. 293-301, 1972.

[24] VIDAURRE, G., LOMBARDI, L. R., OLIVEIRA, J. T. S., et al., "Lenho Juvenil e Adulto e as Propriedades da Madeira", Floresta e Ambiente, v. 18, n. 4, pp. 469-480, 2011.

[25] PElOzZI, M. M. A., SEVERO, E. T. D., CALONEGO, F. W., et al., "Propriedades físicas dos lenhos juvenil e adulto de Pinus elliottii Engelm var. elliottii e de Eucalyptus grandis Hill ex Maiden", Ciência Florestal, v. 22, n. 2, pp. 305-313, 2012.

[26] MORAIS, S. A. L., NASCIMENTO, E. A., MELO, D. C. “Análise da madeira de Pinus oocarpa parte I - estudo dos constituintes macromoleculares e extrativos voláteis", Revista Árvore, v.29, n.3, pp.461-470, 2005.

[27] BARBOSA, L. C. A., MALTHA, C. R. A., CRUZ, M. P. "Composição química de extrativos lipofílicos e polares de madeira de Eucalyptus grandis”, Ciência \& Engenharia, v. 15, n. 2, pp. 13- 20, 2005.

[28] KIM, M., OCHIISHI, M., MATSUMARA, J., et al., "Variation in wood properties of six natural acacia hybrid clones in northern Vietnam", Journal of Wood Science, v. 54, n. 6, pp. 436-442, 2008.

[29] SHEBANI, A. N., REENEN, A. J. V., MEINCKEN, M. "The effect of wood extractives on the thermal stability of different wood species", Thermochimica Acta, v. 471, n. 12, pp. 43-50, 2008.

[30] SHESHMANI, S. "Effects of extractives on some properties of bagasse/high density polypropylene composite", Carbohydrate Polymers, v. 94, n. 1, pp. 416-419, 2013.

[31] PROTÁSIO, T. P., BUfALINO, L., TONOLI, G. H. D., et al., "Brazilian lignocellulosic wastes for bioenergy production: characterization and comparison with fossil fuels", Bioresources, v. 8, n. 1, pp. 1166$1185,2013$.

[32] ZANUNCIO, A. J. V., COLODETTE, J. L., GOMES, F. J. B., et al., "Composição química da madeira de eucalipto com diferentes níveis de desbaste”, Ciência Florestal, v. 23, n. 4, pp. 755-760, 2013. 